\title{
Evaluación de la deflexión de la base del cráneo. Un estudio cefalométrico
}

\author{
Evaluation of the cranial base deflection. A cephalometric study
}

\section{Resumen}

Objetivo: Evaluar la deflexión de la base del cráneo en pacientes con diferente clase esquelética. Materiales y método: El estudio descriptivo y retrospectivo incluyó 318 radiografías cefalométricas de niños peruanos entre 6 y 12 años de edad, pertenecientes a la Facultad de Odontología de la Universidad Nacional Mayor de San Marcos y al Servicio de Ortodoncia y Ortopedia Maxilar del Instituto Nacional de Salud del Niño entre los años 2000 y 2015. Se evaluó la deflexión de la base craneal tomando como referencia el ángulo NSBa, el cual fue comparado con las tres clases esqueléticas tomando como referencia el ángulo ANB. Se evaluó también según sexo y edad. Resultados: El valor de la deflexión de la base craneal fue de $132^{\circ} \pm 4,85^{\circ}$. En el grupo de Clase I fue de $132,23^{\circ} \pm 5^{\circ}$, en el grupo de Clase II: $132,21^{\circ} \pm 4,81^{\circ}$ y en el grupo de Clase III: $130,23^{\circ} \pm 4,29^{\circ}$ (se encontraron diferencias significativas $\mathrm{p}<0,05)$. La deflexión de la base craneal en mujeres fue de $131,9^{\circ} \pm 4,86^{\circ}$ y en varones fue de $132^{\circ} \pm 4,87^{\circ}$ (No se encontraron diferencias significativas $\mathrm{p}=0,831$ ). El valor hallado en los pacientes de 6 ańos de edad fue de $130,80^{\circ} \pm 4,44^{\circ}$ mientras que en los de 12 ańos de edad fue de $129,76^{\circ} \pm 3,05^{\circ}$ (no se encontraron diferencias significativas $\mathrm{p}=0,275$ ). Conclusiones: La deflexión de la base del cráneo es similar en niños peruanos de Clase I y Clase II pero diferente en Clase III.

Palabras clave: Base del Cráneo; Cefalometría; Niños.

\section{Abstract:}

Objective: To evaluate the cranial base deflection in different skeletal class patients. Materials and method: Descriptive and retrospective study included cephalometric radiographs of 318 Peruvian children, with an age range from 6 to 12 years selected from the School of Dentistry at San Marcos University and the Orthodontics and Maxillofacial Orthopedics Department at Children's National Health Institute taken between 2000 and 2015. Cranial base deflection was evaluated taking NSBa angle as a reference and compared with the three skeletal classes taking ANB angle as a reference. Sex and age were also compared. Results: Cranial base deflection value was $132^{\circ} \pm 4.85^{\circ}$. In Class I group it was $132.23^{\circ} \pm 5^{\circ}$, in Class II group: $132.21^{\circ} \pm 4.81^{\circ}$ and in Class III group: $130.23^{\circ} \pm 4.29^{\circ}$ (significant differences were found $p<0.05$ ). Cranial base deflection in females was $131.9^{\circ} \pm 4.86^{\circ}$ and $132^{\circ} \pm 4.87^{\circ}$ in males (no significant differences were found $\mathrm{p}=0.831$ ). In 6 year-old patients the deflection value was $130.80^{\circ} \pm 4.44^{\circ}$ while in the 12 year-old group was $129.76^{\circ} \pm 3.05^{\circ}$ (No significant differences were found $\mathrm{p}=0.275$ ). Conclusions: The cranial base deflection is similar in Class I and Class II Peruvian children but different in Class III.

Keywords: Cranial base; Cephalometry; Children.

\section{Introducción}

La base craneal es la estructura más compleja del esqueleto humano, la cual tiene como función formar la plataforma central sobre la cual se desarrolla el cerebro y la cara ${ }^{1}$. Filogenéticamente, es la parte más primitiva del esqueleto craneofacial y base estructural de la arquitectura craneofacial ${ }^{1}$.
Está compuesta por diferentes huesos (esfenoides, etmoides, frontal, parietal, temporal y occipital) interconectados por las sincondrosis ${ }^{2}$, puede dividirse en dos regiones: la base craneal anterior, que se extiende desde el centro de la fosa hipofisaria a la sutura fronto nasal y la base craneal posterior, se extiende desde el centro de la fosa hipofisaria al margen anterior del agujero magno ${ }^{1}$.

\author{
Adara Flores Angeles ${ }^{1, a}$, Luciano \\ Soldevilla Galarza ${ }^{2, b}$ \\ 1 Facultad de Odontología. Universidad Nacio- \\ nal Mayor de San Marcos. Lima, Perú. \\ 2 Departamento Académico de Estomatología \\ Pediátrica. Facultad de Odontología. Univer- \\ sidad Nacional Mayor de San Marcos. Lima, \\ Perú. \\ a Cirujana Dentista \\ b Especialista en Ortodoncia
}

Correspondencia:

Adara Flores Angeles

Correo electrónico: adiangeles2409@outlook. com

Av. Petit Thouars 1059. Urbanización Santa Beatriz, Lima 1, Perú.

Coautor:

Luciano Soldevilla Galarza

lsoldevillag@unmsm.edu.pe

Conflicto de intereses: Los autores declaran no tener ningún conflicto de interés en la publicación del artículo.

Fuente de financiamiento: Autofinanciado.

Fecha de recepción: 10/06/17

Fecha de aceptación: 28/06/17

La base del cráneo puede asumir dos modalidades de crecimiento: extensión o flexión, que es cuantificado radiográficamente como el ángulo formado por los puntos cefalométricos nasion, sella y basion (NSBa) en el plano sagital medio ${ }^{3}$. Según la literatura este ángulo presenta una gran variación durante el crecimiento fetal y postnatal ${ }^{3}$, siendo así el ángulo al nacer $142,8^{\circ}$ aproxi- 
madamente, a los cinco años de edad se reduce a $130,8^{\circ}$. Manteniéndose relativamente estable entre los cinco a quince años, con una angulación entre $130^{\circ}$ y $135^{\circ}{ }^{4}$. El complejo nasomaxilar se asocia con la base craneal anterior y la mandíbula con la base craneal posterior ${ }^{5}$. Por lo tanto, a partir de esta relación es posible suponer que las variaciones en el crecimiento y orientación de estas regiones pueden alterar las posiciones del maxilar y la mandíbula en relación con la base craneal y entre sí. Esto a su vez puede influir en la clase esquelética y el tipo de maloclusión.

Los estudios realizados en individuos con maloclusión Clase II y Clase III han permitido concluir que las variaciones morfológicas entre estas maloclusiones están asociadas al ángulo de la base del cráneo y que la flexión o extensión de la base craneal puede influir fuertemente en el posicionamiento mandibular, a través del desplazamiento de la cavidad glenoidea. Cuando hay extensión de la base del cráneo se genera una elongación de ésta, incrementándose el ángulo de la base de cráneo, lo que producirá una implantación posterior y superior de la articulación temporomandibular (ATM), facilitando el desplazamiento de la mandíbula a una posición más posterior y junto a otros factores etiopatogénicos se puede orientar hacia el establecimiento de una maloclusión Clase II. Cuando hay flexión de la base craneal se produce una inhibición de la elongación de la base de cráneo, acortándose el ángulo de la base de cráneo, originando una implantación anterior e inferior de la ATM, desplazando la mandíbula a una posición más anterior, este proceso y dependiendo de otros factores genéticos y funcionales se puede orientar el desarrollo de una maloclusión Clase III ${ }^{5}$.

Chin y cols. ${ }^{6}$ indicaron una tendencia de reducción del ángulo de la base craneal progresivamente de Clase II hacia la maloclusión de Clase III. Rizwan y cols. ${ }^{4}$ encontraron una disminución progresiva del ángulo de la base craneal en las maloclusiones Clase II, Clase I y Clase III. Gong y cols. ${ }^{7}$ en un metaanálisis encontraron que los pacientes Clase III presentan un ángulo significativamente menor de la base del cráneo que los pacientes Clase I y Clase II. La relación etiológica entre el crecimiento de la base del cráneo y el tipo de maloclusión sigue siendo controversial, debido a que no todas las investigaciones son concluyentes en asegurar que el grado de flexión o extensión puede afectar la posición de los maxilares y por lo tanto ser un factor fundamental en la determinación del tipo de maloclusión ${ }^{2,8,9}$. Además la mayoría de estos estudios se basan en investigaciones realizadas en poblaciones de origen caucásico, lo cual limita obtener un diagnóstico asertivo, ya que una población peruana mestiza con características raciales muy distintas de la población caucásica, debe tener sus propios valores promedio específicos de la deflexión de la base del cráneo según edad y sexo.

Es por ello, que el propósito de la presente investigación fue evaluar la deflexión de la base del cráneo en pacientes con Clase I, Clase II y Clase III esquelética.

\section{Materiales y método}

Se diseñó un estudio descriptivo y retrospectivo que evaluó radiografías cefalométricas laterales de pacientes peruanos, en crecimiento, con edades entre 6 y 12 ańos. La muestra fue seleccionada en base a un método probabilístico y estratificado a partir de radiografías procedentes de la Facultad de Odontología de la Universidad Nacional Mayor de San Marcos y al Servicio de Ortodoncia y Ortopedia Maxilar del Instituto $\mathrm{Na}$ cional de Salud del Niño entre los años 2000 y 2015, las cuales fueron observadas por un especialista en el área de Ortodoncia; quien estableció un diagnóstico de la clase esquelética; posterior a ello se tomaron en cuenta aquellas en donde exista coincidencia en la clasificación de la clase esquelética dada por el especialistas de Ortodoncia y la operadora. Se asignaron números para cada uno de las radiografías y se procedió a realizar un muestreo aleatorio simple estratificado obteniéndose así 318 radiografías. Para calcular el tamaño de la muestra se consignaron los siguientes datos (en base a los resultados de un estudio piloto): nivel de confianza 95\%, error muestral $1^{\circ}$, el valor obtenido de " $n$ " fue 82 radiografías. Sin embargo se trabajó con una población de 482 radiografías para obtener una muestra representativa de cada clase esquelética. Posteriormente se dividió la población en estratos según clase esquelética y de cada uno se seleccionó una muestra, obteniéndose finalmente: clase I esquelética: 119 radiografías, clase II esquelética: 164 radiografías y clase III esquelética: 35 radiografías.

Se obviaron radiografías de pacientes menores de 6 y mayores de 12 años de edad, con patologías óseas y/o de tejido blando que abarque la zona de los puntos cefalométricos de interés, con distorsiones o alteración de calidad y que hayan sido tomadas antes del año 2000 y después del 2015.

El trazado cefalométrico se realizó siguiendo los contornos óseos y de tejido blando; la ubicación de los puntos cefalométricos analizados se resumen en la figura 1 .

La clase esquelética fue evaluada según el ángulo ANB y catalogada como Clase I cuando el ángulo midiera de $0^{\circ}$ a $4^{\circ}$, Clase II con un ángulo mayor de $4^{\circ}$ y Clase III con un valor menor de $0^{\circ}$. La deflexión de la base craneal fue evaluada según el ángulo NSBa y fue catalogada como extensión de la base craneal cuando el valor fuera mayor de $135^{\circ} \mathrm{y}$ flexión de la base craneal cuando fuera menor de $130^{\circ}$. La edad de los pacientes se evaluó según categorías de $6,7,8$, 9, 10,11 y 12 años.

Los datos fueron recolectados por un observador (AF) quien realizó una calibración inter-observador con un especialista en Ortodoncia (LS); la concordancia entre ambos fue realizada a través de un estudio piloto que incluyó 20 radiografías cefalométricas; el grado de concordancia según el Coeficiente de Correlación Intraclase fue de 0,997 para el ángulo SNBa. La evaluación del error intra-observador se realizó con otras radiografías evaluadas luego de un mes de blanqueo; según la prueba de Dahlberg con un nivel de significancia del 5\%. Encontrándose un valor de error de medición de 0,32 grados. Ambas pruebas dieron como resultado que no existía una diferencia estadísticamente significativa.

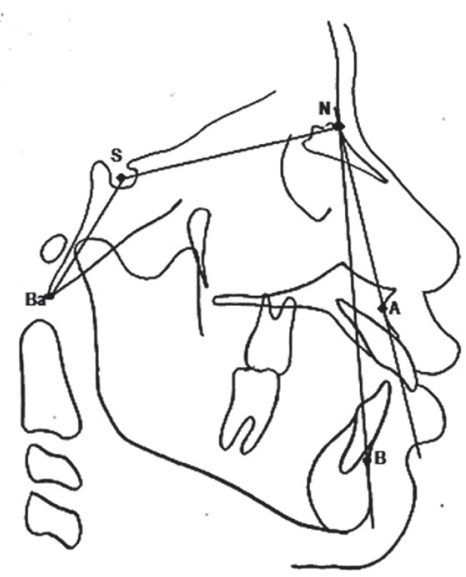

Figura 1. Ángulos analizados en el trazado cefalométrico. ANB: Ángulo subespinal (A)nasion (N)-supramental (B): Ángulo de la relación anteroposterior del maxilar y la mandíbula. NSBa: Ángulo nasion (N)-sella (S)basion (Ba): Ángulo de la base del cráneo. 
Las pruebas de Kolmogorov-Smirnov y Shapiro-Wilk, $(p>0,05)$ determinaron la ausencia de distribución normal de los datos. Las medias de los tres grupos según edad y sexo se compararon utilizando las pruebas de Kruskal-Wallis y U de Mann-Whitney con un nivel de significancia de 5\%. Los datos fueron analizados utilizando el paquete estadístico SPSS versión 19,0.

\section{Resultados}

Al analizar la deflexión de la base del cráneo según clase esquelética se encontró que el ángulo NSBa presentó diferencias estadísticamente significativas en las tres clases esqueléticas; encon-

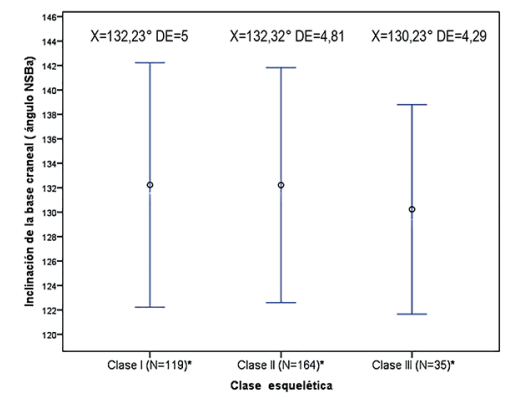

Figura 2. Mediciones de la deflexión de la base craneal según clase esquelética

${ }^{*}$ Clase I, II y III $(\mathrm{p}<0,05)$

** Clase I y III $(\mathrm{p}=0,03)$ y Clase II y III $(\mathrm{p}=0,016)$

*Prueba de Kruskal Wallis

**Prueba de U Mann Whitney

$\mathrm{N}=$ número de casos. $\mathrm{X}=$ Media. $\mathrm{DE}=$ Desviación estándar trándose diferencias entre la Clase III y Clase I, así como entre la Clase III y Clase II (Figura 2).

$\mathrm{Al}$ evaluar según sexo, el ángulo NSBa no presentó diferencias estadísticamente significativas entre las Clases I, II y III en mujeres ni en varones (Figura 3).

En la figura 4 se puede apreciar que, las variaciones de los promedios de deflexión de la base del cráneo en los pacientes del sexo masculino fueron mayores con respecto al sexo femenino de acuerdo a la edad, con excepción de los pacientes de 8, 9 y 10 ańos.

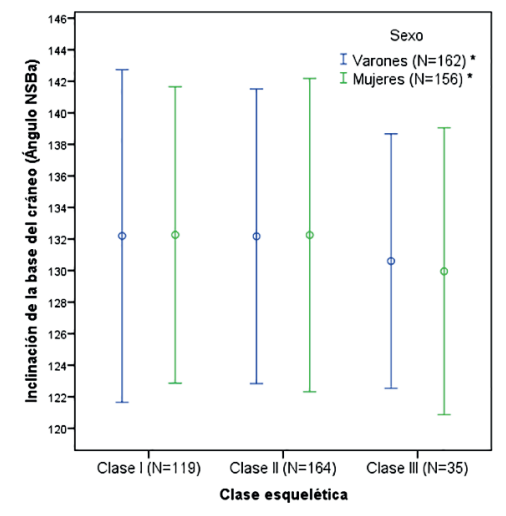

Figura 3. Mediciones de la deflexión de la base craneal según clase esquelética y sexo

*Varones Clase I, II y III ( $\mathrm{p}=0,271)$

${ }^{*}$ Mujeres Clase I, II y III $(\mathrm{p}=0,158)$

${ }^{* *}$ Varones y mujeres $(\mathrm{p}=0,831)$

*Prueba de Kruskal-Wallis

** Prueba U de Mann-Whitney

$\mathrm{N}=$ número de casos.

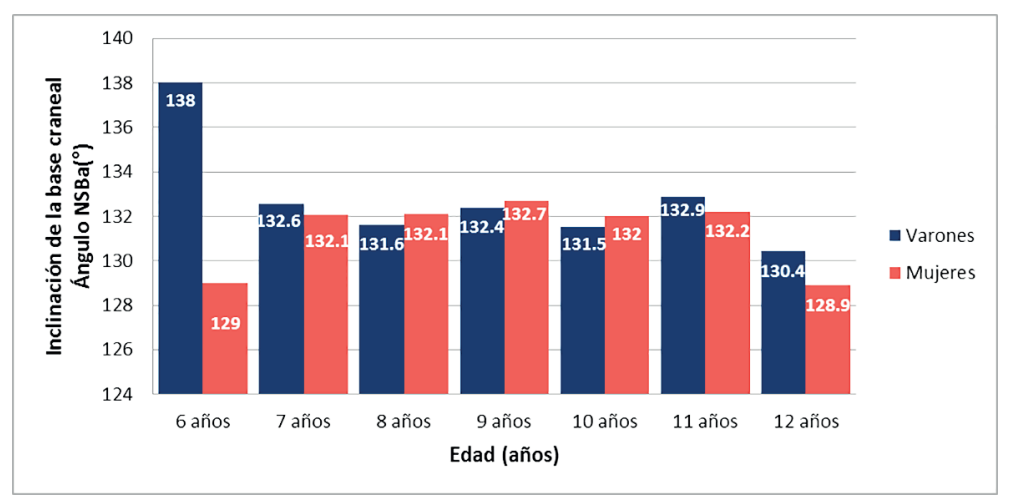

Figura 4. Promedios de la deflexión de la base del cráneo según sexo y edad.

En los resultados con respecto a la edad, los pacientes de Clase I, II y III no mostraron diferencias estadísticamente significativas en relación al ángulo NSBa (Tabla 1).

\section{Discusión}

En la actualidad uno de los principales exámenes auxiliares de diagnóstico sigue siendo la radiografía cefalométrica lateral y aunque es una representación bidimensional de una estructura tridimensional, la interpretación del análisis cefalométrico nos brinda datos importantes para la evaluación del crecimiento de los componentes del complejo craneofacial del paciente, incluyendo a la base del cráneo. La hipótesis de una posible correlación entre el ángulo de la base craneal y el desarrollo de las maloclusiones fue incorporada a principios del siglo XX; desde entonces hasta la actualidad sigue siendo investigada ${ }^{10}$. Estudios realizados por Chin y cols. ${ }^{6}$ y Gong y cols. ${ }^{7}$ sugieren que existe una asociación entre la forma y longitud de la base craneal y la maloclusión esquelética.

Thiesen y cols. ${ }^{2}$ y Chin y cols. ${ }^{6}$ indicaron una tendencia de reducción del ángulo de la base craneal progresivamente de Clase II hacia la maloclusión de Clase III. Shah y cols. ${ }^{4}$ observaron una disminución progresiva del ángulo de la base craneal en las maloclusiones Clase II, Clase I y Clase III. Estos resultados coinciden con los hallazgos de nuestro estudio, donde se confirmó que los pacientes con Clase III esquelética presentaban una flexión de base del cráneo, con diferencias estadísticamente significativas entre el grupo Clase III con Clase I y Clase III con Clase II. Estas observaciones realizadas en individuos con diferentes maloclusiones, nos permiten concluir que las variaciones morfológicas entre Clase II y Clase III están asociadas al ángulo de la base del cráneo, y que la flexión de la base craneal influye en un mayor desplazamiento anterior de la mandíbula mediante la determinación de la posición anteroposterior del cóndilo en relación con el perfil facial, contribuyendo en el desarrollo de una maloclusión Clase III.

Tabla 1. Mediciones de la deflexión de la base craneal $\left(\mathrm{NSBa}^{\circ}\right)$ según clase esquelética en diferentes edades

\begin{tabular}{|c|c|c|c|c|c|c|c|c|c|c|c|c|c|c|c|}
\hline \multirow{2}{*}{ Relación Esquelética } & \multicolumn{2}{|c|}{6 años } & \multicolumn{2}{|c|}{7 años } & \multicolumn{2}{|c|}{8 años } & \multicolumn{2}{|c|}{9 años } & \multicolumn{2}{|c|}{10 años } & \multicolumn{2}{|c|}{11 años } & \multicolumn{2}{|c|}{12 años } & \multirow{2}{*}{$p$} \\
\hline & $x$ & $\mathrm{DE}$ & $x$ & $\mathrm{DE}$ & $x$ & $\mathrm{DE}$ & $x$ & $\mathrm{DE}$ & $x$ & $\mathrm{DE}$ & $x$ & $\mathrm{DE}$ & $x$ & $\mathrm{DE}$ & \\
\hline Clase I & 129,5 & 3,54 & 132,57 & 5,08 & 131 & 4,59 & 134,27 & 4,17 & 131 & 4,59 & 134,27 & 4,17 & 128,75 & 2,49 & 0,13 \\
\hline Clase II & 133,5 & 6,36 & 132,39 & 4,03 & 132,28 & 5,24 & 132,5 & 6,12 & 132,28 & 5,24 & 132,5 & 6,12 & 130,4 & 2,91 & 0,856 \\
\hline Clase III & 128 & & 128,5 & 2,12 & 130,83 & 2,37 & 130,67 & 5,07 & 130,83 & 2,37 & 130,67 & 5,07 & 130,33 & 5,13 & 0,988 \\
\hline Total & 130,8 & 4,44 & 132,3 & 4,58 & 131,76 & 4,99 & 132,6 & 5,36 & 131,76 & 4,99 & 132,6 & 5,36 & 129,76 & 3,05 & 0,275 \\
\hline
\end{tabular}

Prueba de Kruskal-Wallis

$\mathrm{X}=$ Media. $\mathrm{DE}=$ Desviación estándar 
Thiesen y cols. ${ }^{2}$, Chin y cols. ${ }^{6}$, Gong y cols. ${ }^{7}$ y Hassan. ${ }^{11}$ han afirmado que los individuos de Clase II presentan un mayor ángulo de la base craneal, lo que produciría una implantación posterior y superior de la articulación temporomandibular (ATM), ubicando la mandíbula en una posición posterior. Estas investigaciones coinciden con nuestros resultados, corroborando que un mayor ángulo de la base craneal puede orientar el desarrollo de una maloclusión Clase II.

Días y cols. ${ }^{5}$, Chin y cols. ${ }^{6}$, Gong y cols. ${ }^{7}$, Flórez ${ }^{8}$, Sundareswaran ${ }^{12}$ y Sanggarnjanavanich y cols. ${ }^{13}$ han reportado ángulos más agudos en la morfología de los pacientes Clase III lo que originaría una implantación más anterior e inferior de la cavidad glenoidea y de la ATM, desplazando la mandíbula a una posición más anterior. Estos resultados coindicen con los hallazgos del presente estudio, reafirmando que la flexión de la base craneal, puede orientar el establecimiento de una maloclusión Clase III. Sin embargo, Thiesen y cols. ${ }^{2}$, Flórez ${ }^{8}$, Hassan ${ }^{12}$ y Cossio y cols. ${ }^{14}$ reportaron diferencias no significativas entre los individuos del grupo Clase I y Clase II. Del mismo modo, en la presente investigación no se encontró diferencias estadísticamente significativas en el ángulo de la base craneal de los grupos con Clase I y Clase II esquelética, esto se debe posiblemente a los rasgos estructurales intrínsecos de los pacientes del grupo Clase I, que han compensado en mayor o menor grado las tendencias del crecimiento de la base del cráneo mencionadas anteriormente.

No obstante, la relación etiológica entre la flexión de base craneal y el tipo de maloclusión sigue siendo controversial, debido a que no todas las investigaciones concluyen en asegurar que el grado de flexión o extensión puede afectar la posición de los maxilares, por lo tanto consideran que la base del cráneo no ejerce un rol fundamental en el establecimiento de las diferentes maloclusiones ${ }^{4,8,9,15}$.

Los resultados del estudio indicaron que existe una amplia gama de variaciones en valores del ángulo de la base del cráneo en niños peruanos. El promedio del ángulo NSBa osciló entre $128^{\circ}$ y $134,27^{\circ}$. Esta diferencia con respecto al "gold estándar", puede ser el resultado de la edad, las características propias de nuestra población y la amplia variedad de grupos raciales. Por otro lado, la flexión de la base del cráneo y las diferencias estadísticamente significativas en niños con Clase III esquelética, reafirma que estos pacientes presentan una menor deflexión de la base del cráneo.
Se destaca la importancia de considerar la deflexión de la base del cráneo en el diagnóstico, considerando el rol de la base del cráneo en la evaluación ortodóntica de la discrepancia esquelética anteroposterior de los maxilares.

\section{Conclusiones}

La deflexión de la base craneal de la muestra estudiada fue $132^{\circ} \pm 4,85^{\circ}$, considerada como una deflexión dentro del "gold estándar".

Los pacientes con Clase III esquelética obtuvieron el menor valor de deflexión de base craneal y presentaron diferencias estadísticamente significativas con los pacientes Clase I y Clase II esquelética; y dentro de este grupo, fueron las mujeres y los pacientes de 6 años de edad, los que presentaron valores menores de deflexión de la base craneal.

Los pacientes Clase I y Clase II esquelética no presentaron diferencias significativas en la deflexión de la base del cráneo.

La flexión y extensión de la base del cráneo, junto a otros factores etiopatogénicos pueden influir de manera significativa en el desarrollo de las clases esqueléticas.

\section{Referencias bibliográficas}

1. Lieberman DE. The evolution of the human head. 1st ed. London: Belknap Press. 2011.

2. Thiesen G, Pletsch G, Zastrow MD, Martins do Valle CV, Martins do Valle K, Paim $M$ et al. Comparative analysis of the anterior and posterior length and deflection angle of the cranial base, in individuals with facial pattern I, II and III. Dental Press J Orthod. 2013;18(1):69-75.

3. Sperber GH, Sperber SM, Guttman GD. Craniofacial Embryogenetics and Development. 2nd ed. Shelton: People's Medical Publishing House USA. 2010.

4. Shah R, Mushtaq M, Mahmood A. The relationship between cranial base angle and various malocclusion types. Pak Orthod J. 2015;7(1):8-12.

5. Días OP, Marchioro EM, Rizzato S, Santayana de Lima EM. Comparative study of linear and angular measures of the cranial base in skeletal Class I and III malocclusion. Rev Odonto Cienc. 2011;26(2):126-132.
6. Chin A, Perry S, Liao C, Yang Y. The relationship between the cranial base and jaw base in Chinese population. Head Face Med. 2014;10(1):31. DOI: 10.1186/1746-160X-10-31

7. Gong A, Lib J, Wangc Z, Lid Y, Huc F, Lic Q et al. Cranial base characteristics in anteroposterior malocclusions: A meta-analysis. Angle Orthod. 2015;86(4):1-13. DOI: $10.2319 / 032315-186.1$

8. Flórez CV, Arriola GL. Evaluación cefalométrica de la longitud y deflexión craneal en sujetos con diferente patrón de crecimiento. Rev Ort AIO-Perú. 2014;01(1):21-28.

9. Dhopatkar A, Bhatia S, Rock P. An investigation into the relationship between the cranial base angle and malocclusion. Angle Orthod. 2002;72(5):456-463.

10. Brodie AG. The behavior of the cranial base and its components as revealed by serial cephalometric roentgenograms. Angle Orthod. 1955;25(1):148-160.

11. Hassan AH. Cephalometric characteristics of Class II division 1 malocclusion in a Saudi population living in the western region. Saudi Dent J. 2011;23(1):23-27.

12. Sundareswaran S, Thirumoorty SN. Anterior cranial base features in skeletal Class III patients with maxillary recession: a cephalometric study. Orthodontics (Chic.). 2012;13(1):105-115.

13. Sanggarnjanavanich S, Sekiya T, Nomura Y, Nakayama T, Hanada $\mathrm{N}$, Nakamura Y. Cranial base morphology in adults with skeletal Class III malocclusion. Am J Orthod Dentofacial Orthop. 2014;146(1):82-91. DOI: 10.1016/j.ajodo.2014.04.014

14. Cossio L, López J, Rueda Z, Botero P. Morphological configuration of the cranial base among children aged 8 to 12 years. BMC Res Notes. 2016;9:309. DOI: 10.1186/s13104-016-2115-2.

15. Bilal I, Fakhri A. Cranial base morphology in different skeletal clases (a cross- sectional lateral cephalometric study). J Bagh Coll Dentistry. 2013;25(1):108-13. 